PROCEEDINGS OF THE

AMERICAN MATHEMATICAL SOCIETY

Volume 134, Number 11, November 2006, Pages 3153-3157

S 0002-9939(06)08683-7

Article electronically published on June 5, 2006

\title{
THE WEAK DIRICHLET PROBLEM FOR BAIRE FUNCTIONS
}

\author{
JIŘÍ SPURNÝ \\ (Communicated by Jonathan M. Borwein)
}

\begin{abstract}
Let $X$ be a simplex and $K$ a compact subset of the set of all extreme points of $X$. We show that any bounded function $f$ of Baire class $\alpha$ on $K$ can be extended to a function $h$ of affine class $\alpha$ on $X$. Moreover, $h$ can be chosen in such a way that $h(X) \subset \overline{\mathrm{co}} f(K)$.
\end{abstract}

\section{INTRODUCTION}

If $X$ is a simplex in a locally convex space (for the notions not explained here we refer reader to the next section) and $K$ is a compact subset of the set ext $X$ of all extreme points of $X$, it is well known that any continuous function on $K$ can be extended to an affine continuous function on $X$ (see [1, Theorem II.3.12] or 2, Corollary 7.7]). The problem of finding an affine extension for a function defined on a compact subset of ext $X$ is sometimes called the weak Dirichlet problem. In our paper we investigate this question for Baire functions.

We remark that the general question of finding an affine extension for a function defined on a compact subset of ext $X$ is of some interest. Our Corollary 3.5 serves as an example of a purely topological application of "affine extension theorems".

Let $\mathcal{A}(X)$ stand for the space of all affine continuous functions on $X$. We set $\mathcal{B}_{0}^{b b}(\mathcal{A}(X)):=\mathcal{A}(X)$ and having $\mathcal{B}_{\beta}^{b b}(\mathcal{A}(X)), \beta<\alpha$, already defined for an ordinal number $\alpha \in\left(0, \omega_{1}\right)$, we set $\mathcal{B}_{\alpha}^{b b}(\mathcal{A}(X))$ to be the space of all pointwise limits of bounded sequences of functions from $\bigcup_{\beta<\alpha} \mathcal{B}_{\beta}^{b b}(\mathcal{A}(X))$. We say that a function $h \in \mathcal{B}_{\alpha}^{b b}(\mathcal{A}(X))$ is of affine class $\alpha$ (see [3]). With this notation we can formulate the main result of the paper.

Theorem 1.1. Let $X$ be a simplex and let $K \subset$ ext $X$ be compact. Then for any bounded function $f$ on $K$ of Baire class $\alpha$ there exists a function $h \in \mathcal{B}_{\alpha}^{b b}(\mathcal{A}(X))$ such that $h=f$ on $K$ and $h(X) \subset \overline{\mathrm{co}} f(K)$.

Received by the editors January 25, 2005.

2000 Mathematics Subject Classification. Primary 46A55; Secondary 26A21.

Key words and phrases. Simplex, weak Dirichlet problem, affine functions, Baire functions.

The author is currently a Postdoctoral Fellow at the Department of Mathematical and Statistical Sciences of the University of Alberta, Edmonton. He would like to thank this department and, in particular, Prof. N. Tomczak-Jaegermann and Prof. V. Zizler for support and excellent working conditions.

This research was supported in part by the grants GA ČR 201/03/0935, GA ČR 201/03/D120, NSERC 7926, and in part by the Research Project MSM 0021620839 from the Czech Ministry of Education. 
We remark that the proof of Theorem 1.1 is easy if $K$ is metrizable since we are able to use almost straightforwardly Lazar's selection theorem [5, Theorem 3.1]. We sketch the proof of this observation in Remark 3.4. However, for nonmetrizable sets we have to apply the above-mentioned Lazar's selection theorem more carefully as we explain below.

\section{Preliminaries}

All topological spaces will be considered as Hausdorff. If $X$ is a compact space, we denote by $\mathcal{C}(X)$ the space of all continuous functions on $X$. Let $\mathcal{M}^{1}(X)$ denote the set of all probability Radon measures on $X$ and let $\varepsilon_{x}$ stand for the Dirac measure at $x \in X$.

If $\mathcal{F}$ is a family of functions on a set $X$, we write $(\mathcal{F})_{1}$ for the family of all pointwise limits of sequences of functions from $\mathcal{F}$. We set $\mathcal{B}_{0}(\mathcal{F}):=\mathcal{F}$ and inductively define $\mathcal{B}_{\alpha}(\mathcal{F}):=\left(\bigcup_{\beta<\alpha} \mathcal{B}_{\beta}(\mathcal{F})\right)_{1}$ for each $\alpha \in\left(0, \omega_{1}\right)$. The family consisting of all pointwise limits of bounded sequences of functions from $\mathcal{F}$ is denoted as $(\mathcal{F})_{1}^{b b}$. Similarly as above we define $\mathcal{B}_{\alpha}^{b b}(\mathcal{F})$ for $\alpha \in\left[0, \omega_{1}\right)$.

If $X$ is a topological space and $\alpha \in\left(0, \omega_{1}\right)$, we write $\mathcal{B}_{\alpha}(X)$ for the space of all Baire functions of class $\alpha$ on $X$, i.e., $\mathcal{B}_{\alpha}(X)=\mathcal{B}_{\alpha}(\mathcal{C}(X))$. It is easy to see that any bounded function of Baire class $\alpha$ belongs to $\mathcal{B}_{\alpha}^{b b}(\mathcal{C}(X))$.

Let $X$ be a compact convex subset of a locally convex space. Given a point $x \in X$, we say that a measure $\mu \in \mathcal{M}^{1}(X)$ represents $x$ if $\mu(h)=h(x)$ for every $h \in \mathcal{A}(X)$. The set ext $X$ of all extreme points consists precisely of those points $x \in X$ for which there exists no representing measure except $\varepsilon_{x}$ (see [1, Corollary I.2.4] or [2, Theorem 6.3]).

A convex set $F \subset X$ is called a face if each open segment $I \subset X$ intersecting $F$ is contained in $F$.

The convex cone of all continuous convex functions on $X$ determines a partial ordering $\preceq$ (called the Choquet ordering) on the space $\mathcal{M}^{+}(X)$ of all positive Radon measures on $X: \mu \preceq \nu$ if and only if $\mu(f) \leq \nu(f)$ for each convex continuous function $f$ on $X$. According to the famous Choquet-Bishop-de-Leeuw theorem (see [1, Theorem I.4.8] or [2, Theorem 6.8]), for every $x \in X$ there exists a maximal (with respect to the Choquet ordering) measure $\mu$ representing $x$. If this maximal measure is uniquely determined for every $x \in X$, we say that $X$ is a simplex (respectively Choquet simplex, see [1, Chapter II., §3] or [2, Section 2.7]). In this case we denote the unique maximal measure for $x$ as $\delta_{x}$.

If $f$ is a bounded Borel function on a Borel subset $B$ of simplex $X$, we define the function

$$
H^{f}(x):=\delta_{x}(f), \quad x \in X,
$$

where $f$ is defined by 0 on $X \backslash B$.

If $F$ is a subset of a locally convex space, we denote by $\overline{c o} F$ the closed convex hull of $F$. If $X$ is a set, the restriction of a function $f: X \rightarrow \mathbb{R}$ to a set $F \subset X$ is denoted by $f \uparrow_{F}$.

\section{Proof of Theorem 1.1}

Unless otherwise stated, $X$ is assumed to be a simplex in some locally convex space. 
Lemma 3.1. Let $\mathcal{H}=\left\{f_{n}: n \in \mathbb{N}\right\}$ be a bounded family of affine continuous functions on a closed face $F \subset X$. Then there exist a family $\mathcal{A}=\left\{h_{n}: n \in \mathbb{N}\right\}$ of affine continuous functions on $X$ and a mapping $r: X \rightarrow F$ such that $r(x)=x$ on $F$ and $h_{n}(x)=f_{n}(r(x))$ for all $n \in \mathbb{N}$.

Proof. Let $\mathcal{H}$ be as in the premise. Without loss of generality we may assume that $f_{n}(F) \subset[0,1]$ for all $n \in \mathbb{N}$. We consider a mapping $\varphi: F \rightarrow[0,1]^{\mathbb{N}}$ (the space $[0,1]^{\mathbb{N}}$ is considered with the pointwise topology) defined as

$$
x \mapsto\left\{f_{n}(x)\right\}_{n \in \mathbb{N}}, \quad x \in F .
$$

Then $\varphi$ is continuous and affine on $F$. We define a multivalued map $\Phi: X \rightarrow[0,1]^{\mathbb{N}}$ as

$$
\Phi(x):= \begin{cases}\varphi(x) & \text { if } x \in F, \\ \varphi(F) & \text { if } x \in X \backslash F .\end{cases}
$$

Then $\Phi$ has nonempty convex compact values and $\Phi$ is affine, i.e., for every $x, y \in X$ and $\alpha \in[0,1]$,

$$
\alpha \Phi(x)+(1-\alpha) \Phi(y) \subset \Phi(\alpha x+(1-\alpha) y) .
$$

(Here we use the assumption that $F$ is a face.) Since $\Phi=\varphi$ on a closed set $F$ and $\Phi=\varphi(F)$ on the complement of $F$, it is easy to verify that $\Phi$ is even lower semicontinuous, i.e., given an open set $U \subset[0,1]^{\mathbb{N}}$, the set

$$
\Phi^{-1}(U)=\{x \in X: \Phi(x) \cap U \neq \emptyset\}
$$

is open in $X$.

Let $\rho$ be an invariant metric on $\mathbb{R}^{\mathbb{N}}$ which is compatible with the pointwise topology on $[0,1]^{\mathbb{N}}$. Although $\left(\mathbb{R}^{\mathbb{N}}, \rho\right)$ is not complete, its completion $Y$ is a Fréchet space and $[0,1]^{\mathbb{N}}$ is a compact and consequently closed subset of $Y$.

Now we are ready to use Lazar's selection theorem (see [5, Theorem 3.1]) to obtain an affine continuous mapping $\psi: X \rightarrow[0,1]^{\mathbb{N}}$ such that $\psi(x) \in \Phi(x)$ for all $x \in X$.

Set $h_{n}:=\pi_{n} \circ \psi, n \in \mathbb{N}$, where $\pi_{n}:[0,1]^{\mathbb{N}} \rightarrow \mathbb{R}$ is the projection to the $n$-th coordinate. Clearly, each $h_{n}$ is a continuous affine function on $X$.

Since $\psi=\varphi$ on $F, h_{n} \uparrow_{F}=f_{n} \uparrow_{F}$ for all $n \in \mathbb{N}$. If $x \notin F, \psi(x)=\varphi(y)$ for some $y \in F$. If we denote this $y$ as $r(x)$ and set $r(x):=x$ on $F$, we obtain the sought mapping $r$ and conclude the proof.

Lemma 3.2. In the situation of Lemma 3.1, for every $f \in \mathcal{B}_{\alpha}(\mathcal{H})$ there exists a function $h \in \mathcal{B}_{\alpha}(\mathcal{A})$ such that $h(x)=f(r(x))$ for all $x \in X$.

Proof. If $\alpha=0$, i.e, $f=f_{n}$ for some $n \in \mathbb{N}$, take $h:=h_{n}$.

Suppose that the lemma has been verified for all $\beta<\alpha$ where $\alpha \in\left(0, \omega_{1}\right)$. Pick $f \in \mathcal{B}_{\alpha}(\mathcal{H})$. Then there exist functions $f_{n} \in \mathcal{B}_{\alpha_{n}}(\mathcal{H}), n \in \mathbb{N}$, where $\alpha_{n}<\alpha$, such that $f_{n} \rightarrow f$ on $F$. According to the induction hypothesis, for every $n \in \mathbb{N}$ we are able to select $h_{n} \in \mathcal{B}_{\alpha_{n}}(\mathcal{A})$ such that $h_{n}(x)=f_{n}(r(x))$ for every $x \in X$. Since $f_{n}(r(x)) \rightarrow f(r(x))$, the function $h:=\lim _{n} h_{n}$ is well defined, $h \in \mathcal{B}_{\alpha}(\mathcal{A})$ and $h(x)=f(r(x))$ for all $x \in X$. This finishes the proof.

Lemma 3.3. Let $\mathcal{F}$ be a bounded family of continuous functions on a compact set $K \subset \operatorname{ext} X$. Set $F:=\overline{\operatorname{co}} K$.

(i) The set $F$ is a closed face of $X$ and $\operatorname{ext} F=K$. 
(ii) The family $\mathcal{H}:=\left\{H^{f} \uparrow_{F}: f \in \mathcal{F}\right\}$ consists of affine continuous functions on $F$.

(iii) If $f \in \mathcal{B}_{\alpha}(\mathcal{F})$, then $H^{f}{ }_{{ }_{F}} \in \mathcal{B}_{\alpha}(\mathcal{H})$.

Proof. First note that ext $F=K$ due to the Milman theorem (see [2, Theorem 1.3]). According to [2, Lemma 1.6], $F$ is a closed face of $X$ and, consequently, it is a simplex. Since ext $F$ is a closed set, the mapping $x \mapsto \delta_{x}, x \in F$, is continuous on $F$ (see [1, Theorem II.4.1]). From this fact assertion (ii) follows as well as (iii) after a use of transfinite induction.

Proof of Theorem 1.1, By setting $F:=\overline{\mathrm{co}} K$ we obtain a closed face in $X$ (see Lemma 3.3 (i)). Given a function $f \in \mathcal{B}_{\alpha}^{b b}(K)$, find a countable family $\mathcal{F}:=\left\{f_{n}\right.$ : $n \in \mathbb{N}\}$ of continuous functions on $K$ such that $f \in \mathcal{B}_{\alpha}(\mathcal{F})$ and $f_{n}(K) \subset \overline{\operatorname{co}} f(K)$ for every $n \in \mathbb{N}$.

Define $g_{n}:=\left.H^{f_{n}}\right|_{F}, n \in \mathbb{N}$. According to Lemma 3.3 (ii), the family $\mathcal{H}:=$ $\left\{g_{n}: n \in \mathbb{N}\right\}$ consists of continuous affine functions on $F$. Moreover, the values of every function in $\mathcal{H}$ are contained in $\overline{\mathrm{co}} f(K)$. Lemma 3.1 provides a family $\mathcal{A}=\left\{h_{n}: n \in \mathbb{N}\right\}$ of continuous affine functions on $X$ and a mapping $r: X \rightarrow F$ such that $r(x)=x$ on $F$ and $h_{n}(x)=g_{n}(r(x))$ for each $x \in X$ and $n \in \mathbb{N}$. Since $\left.H^{f}\right|_{F} \in \mathcal{B}_{\alpha}(\mathcal{H})$ due to Lemma 3.3 (iii), an application of Lemma 3.2 finishes the proof.

Remark 3.4. We briefly indicate an easier proof of Theorem 1.1 if $K$ is supposed to be metrizable. In this case, $F:=\overline{\mathrm{co}} K$ is a closed face of $X$ (see Lemma 3.3 (i)) which is moreover metrizable.

Indeed, if $b$ is the mapping assigning to each probability measure $\mu$ on $K$ its barycenter (i.e., $b(\mu)$ is the unique point in $X$ such that $\mu$ represents $b(\mu)$ ), then $b$ is a continuous mapping of a metrizable compact set $\mathcal{M}^{1}(K)$ of all probability measures on $K$ onto $F$. Since a continuous image of a compact metric space is metrizable (see [4, Chapter 4, Problem S]), the claim is proved.

Another corollary of Lazar's selection theorem (see [5. Theorem 3.6]) provides an affine continuous retraction $r: X \rightarrow F$. Thus the sought extension of a given function $f \in \mathcal{B}_{\alpha}^{b b}(K)$ can be defined as $\left.H^{f}\right|_{F} \circ r$.

Corollary 3.5. Let $K$ be a closed subset of a compact space $X$. Then for every bounded function $f$ on $K$ of Baire class $\alpha$ there exists a bounded function $h$ on $X$ of Baire class $\alpha$ such that $f=h$ on $K$ and $h(X) \subset \overline{c o} f(K)$.

Proof. If $X$ is a compact space, the set $Y:=\mathcal{M}^{1}(X)$ of all probability measures on $X$ endowed with the weak-star topology is a simplex (see [1, Corollary II.4.2] or [2, Theorem 7.5]). The canonical embedding $\varepsilon: x \mapsto \varepsilon_{x}, x \in X$, serves as a homeomorphism of $X$ onto ext $Y$.

Given a bounded function $f$ on $K$ of Baire class $\alpha$, Theorem 1.1 provides a function $h \in \mathcal{B}_{\alpha}^{b b}(\mathcal{A}(Y))$ such that $h=f \circ \varepsilon^{-1}$ on $\varepsilon(K)$ and $h(Y) \subset \overline{\mathrm{co}}\left(f \circ \varepsilon^{-1}\right)(\varepsilon(K))$. Then the function $h \circ \varepsilon$ is the sought extension of $f$ and we are done.

\section{REFERENCES}

[1] E. M. Alfsen, Compact convex sets and boundary integrals, Springer-Verlag, 1971. MR0445271 $(56: 3615)$

[2] L. Asimow and A.J. Ellis, Convexity theory and its applications in functional analysis, Academic Press, 1980. MR0623459 (82m:46009) 
[3] M. Capon, Sur les fonctions qui vérifient le calcul barycentrique, Proc. London Math. Soc. 32 (1) (1976), 163-180. MR0394148 (52:14952)

[4] R. Engelking, General topology, Verlag, Berlin, 1989. MR.1039321 (91c:54001)

[5] A. Lazar, Spaces of affine continuous functions on simplexes, Trans. Amer. Math. Soc. 134 (1968), 503-525. MR0233188(38:1511)

Faculty of Mathematics and Physics, Charles University, Sokolovská 83, 18675 Praha 8, Czech Republic

E-mail address: spurny@karlin.mff.cuni.cz 